\title{
Direct-acting antiviral therapy for hepatitis C: The initial experience of the University of Cape Town/ Groote Schuur Hospital Liver Clinic, South Africa
}

\author{
M W Sonderup, ${ }^{1}$ B Pharm, MB ChB, FCP (SA), MMed (Medicine), FRCP (Lond); N Gogela, ${ }^{1}$ MB ChB, FCP (SA), MPhil; \\ R Nordien, ${ }^{1}$ MB ChB, FCP (SA), MMed (Medicine); H Smuts, ${ }^{2,3}$ PhD; S Korsman, ${ }^{2,3}$ MB ChB, FC Path (SA) Viro, MMed (Virol Path); \\ D Hardie, ${ }^{2,3}$ MB ChB, MMedPath (Med Virol); C W Spearman, ${ }^{1}$ MB ChB, FCP (SA), MMed (Medicine), PhD, FRCP (Lond) \\ ${ }^{1}$ Division of Hepatology, Department of Medicine, Faculty of Health Sciences, University of Cape Town and Groote Schuur Hospital, Cape Town, \\ South Africa \\ ${ }^{2}$ Medical Virology, National Health Laboratory Service, Groote Schuur Hospital, Cape Town, South Africa \\ ${ }^{3}$ Division of Medical Virology, Department of Pathology, Faculty of Health Sciences, University of Cape Town, South Africa
}

Corresponding author: MW Sonderup (msonderup@samedical.co.za)

\begin{abstract}
Background. An estimated 600000 South Africans are chronically infected with hepatitis C virus (HCV). To date, accurate prevalence data are lacking, but emerging data suggest a significant burden in key populations. Historically, pegylated interferon and ribavirin treatment was challenging, with access limited. The advent of all-oral, short-course direct-acting antiviral (DAA) therapy has revolutionised the management of $\mathrm{HCV}$, being well tolerated and highly effective, although initial cost was a prohibitive factor.

Objectives. To report our initial 2-year experience with DAA therapy at the University of Cape Town/Groote Schuur Hospital Liver Clinic, South Africa (SA).

Methods. Patients who were viraemic for HCV were offered access to DAA therapy. All relevant demographic, virological, serological and clinical laboratory data were captured in a registry. Liver fibrosis was assessed non-invasively with the FibroScan. DAA regimens were prescribed according to current guidance based on HCV genotype (GT), prior treatment history and degree of fibrosis. On treatment, virological response was recorded and a sustained virological response (SVR) was defined as an undetectable HCV RNA at least 12 weeks after the end of treatment.

Results. We report on the first 210 patients treated. Their median (interquartile range (IQR)) age was $52(42-61)$ years and $65 \%$ were male, with men significantly younger than women at $50(42-59)$ years v. $58(47-67)$ years, respectively $(p=0.001)$. All GTs were observed, with 1 and 5 most prevalent at $45 \%$ and $20 \%$, respectively, and GTs 2,3 and 4 frequencies of $7 \%, 11 \%$ and $17 \%$, respectively. Extensive subtype diversity for GTs 2 and 4 was present. The median (IQR) HCV viral $\operatorname{load}_{\text {was }} \log _{10} 5.9 \mathrm{IU} / \mathrm{mL}$ (5.4 - 6.5). A significant proportion of patients (39\%) had advanced fibrosis or cirrhosis, with $11 \% \mathrm{~F} 3$ fibrosis and $28 \% \mathrm{~F} 4$. Of those with cirrhosis, $12 \%$ were decompensated with ChildsPugh B or C disease. Of the patients, $19 \%$ were HIV co-infected and $2 \%$ HBV co-infected. In total, 13\% were treatment experienced. The majority of patients were treated with sofosbuvir and ledipasvir (38\%), daclatasvir (36\%) or velpatasvir ( \pm voxilaprevir, $9 \%$ ). Less frequent combinations included partitaprevir, ritonavir, ombitasvir \pm dasbuvir (11\%) and sofosbuvir/ribavirin (5\%). The per-protocol SVR was $96 \%$ ( $98 \%$ if sofosbuvir/ribavirin is excluded). The majority of treatment failures occurred with GT-4, notably subtype 4 r. Mild side-effects were reported in $10 \%$ of patients, with none discontinuing therapy.

Conclusions. DAA therapy for HCV in a pan-genotypic group of patients, many with advanced liver disease, was highly effective. Our outcomes correspond with existing trial and real-world data for similar treatment. DAA therapy and access need rapid upscaling in SA, especially targeting key populations at point of care.
\end{abstract}

S Afr Med J 2020;110(2):112-117. https://doi.org/10.7196/SAMJ.2020.v110i2.14195

Globally, 71 million people are actively viraemic with the hepatitis C virus (HCV), with an estimated 10.15 million chronically infected in sub-Saharan Africa. ${ }^{[1]}$ An estimated 600000 South Africans have chronic hepatitis $\mathrm{C}$, although the uncertainty intervals are wide. ${ }^{[2]}$ Concomitantly, accurate prevalence data in the general population are generally lacking, although important data are emerging with regard to a high prevalence in high-risk or key populations. ${ }^{[3]}$ The seroprevalence in the general population is thought to be $<1 \%$, and data from the blood transfusion services consistently point towards a low seroprevalence. ${ }^{[4]}$ Key risk populations include people who inject/injected drugs (PWID), men who have sex with men (MSM) and the incarcerated population. A prospective study in HIV-positive MSM in Cape Town noted a hepatitis C seroprevalence of 5.6\% and $0.5 \%$ in the MSM and non-MSM groups, respectively. ${ }^{[5]}$ In a smaller retrospective Cape Town study, 27\% of injecting MSM were HCV antibody-positive; $37.5 \%$ were HIV co-infected. ${ }^{[6]}$ More recently, a large countrywide seroprevalence study of key populations found a $45 \%$ seroprevalence of HCV in PWID; $24.7 \%$ were HIV co-infected. ${ }^{[3]}$ Of MSM, 2.7\% were anti-HCV-positive and 40\% HIV co-infected. Notably, HCV seroprevalence among sex workers was extremely low at $0.1 \%$, but HIV and hepatitis B rates were high.

2019 marked 30 years since HCV was sequenced and cloned for the first time in $1989 .{ }^{[7]}$ In 1991, 3 - 5 times weekly subcutaneous interferon for $\mathrm{HCV}$ demonstrated poor outcomes. Adding ribavirin improved response rates. In 2001, weekly pegylated interferon (PegIFN) plus ribavirin showed significantly improved outcomes. ${ }^{[8,9]}$ Overall, the achievement of a sustained virological response (SVR), defined as the absence of detectable HCV RNA at least 24 weeks 
after the end of treatment, ranged between $45 \%$ and $70 \%$. SVR, an effective cure, was highly dependent on the HCV genotype (GT), in addition to other virological and patient factors. Interferon was contraindicated in many patients, and this, in addition to the cost and often 48-week duration of therapy, with significant adverse effects, made access to treatment impossible for many. The discovery of the HCV replicon system by Bartenschlager and colleagues ${ }^{[10,11]}$ paved the way for the rapid development of new therapies. The 2006 modification of the HCV replicon system heralded a revolution in therapeutic development, culminating in the first all-oral highly effective therapy for HCV, registered in 2014. ${ }^{[12]}$ The targets of the three classes of direct-acting antivirals (DAAs) include the viral nonstructural components NS3/4A protease, NS5A replicase and NS5B polymerase. ${ }^{[13]}$ Combinations of these DAAs, administered for 8 or 12 weeks with or without ribavirin, effectively produce SVR rates well in excess of $90 \%$. In most DAA registration studies, SVR rates exceed 95\%. Treatment is simple and well tolerated and has fundamentally changed the management of hepatitis $\mathrm{C}$, allowing expanded access to previous categories of patients regarded as difficult or not possible to treat with Peg-IFN and ribavirin-based therapy. ${ }^{[14]}$

Initially, however, cost was a major factor, denying access to most. In South Africa (SA), access through a variety of mechanisms early on, albeit on a limited basis, has been possible. ${ }^{[15]}$

\section{Objectives}

To report our initial experience in patients with chronic hepatitis $C$ infection treated with DAA therapy.

\section{Methods}

Patients with proven HCV infection and referred to the Liver Clinic at the University of Cape Town and Groote Schuur Hospital were prospectively captured in a registry in terms of all relevant demographic, serological and virological data. All patients were screened with a laboratory-based anti-HCV antibody test and if positive had active viraemia confirmed with an HCV quantitative viral load (VL) (Cobas Amplicor (Roche, Switzerland) or Xpert (Cepheid, USA)). In those with confirmed viraemia, genotyping was performed with NS5B amplification, sequencing and analysis using the geno2pheno online algorithm (https://www.geno2pheno.org). Additionally, all patients were screened for HIV (if not known to be HIV-positive) and hepatitis B surface antigen (HBsAg; Roche, Switzerland). HIV-infected patients were required to be stable on antiretroviral therapy with a suppressed HIV VL to be eligible for DAA therapy. In those positive for HBsAg, hepatitis B e antigen ( $\mathrm{HBeAg}$ ) and hepatitis B VL (Roche) were measured to assess HBV replication. HIV-negative patients who were HBV co-infected were placed on either lamivudine or tenofovir, with DAA therapy only initiated once HBV VL was undetectable. As a routine, all patients had baseline renal and liver function tests, including an alpha-fetoprotein (AFP) level. At baseline, all patients had hepatitis $\mathrm{C}$ viral sequencing performed to evaluate whether preexisting resistance-associated substitutions (RASs) or variants were present. Standard population-based Sanger sequencing was used. While on treatment, repeat HCV VL, alanine transaminase (ALT) and creatinine were assessed at 4 weeks and at the end of treatment. SVR was assessed by the absence of detectable HCV RNA at least 12 weeks after the end of treatment (SVR12).

Liver fibrosis assessment was performed using vibration-controlled transient elastography (FibroScan; Echosens, France) to acquire a $\mathrm{kPa}$ liver stiffness score, and patients were classified as having F0, F1, F2, F3 or F4 fibrosis. Pretreatment ultrasound screening for hepatocellular carcinoma (HCC) was performed in all patients.
Access to DAA therapy was achieved through various sources. These included: (i) a compassionate use programme from AbbVie Pharma for paritaprevir/ritonavir/ombitasvir/dasabuvir for patients with HCV GT-1 or GT-4 infections; (ii) a donation of sofosbuvir and daclatasvir from Médecins Sans Frontières; (iii) the Groote Schuur Hospital pharmacy, by utilising the existing budget for Peg-IFN/ribavirin for the procurement of DAA therapies; and (iv) patients privately funded or paying out of pocket. Therapies included originator and, where available, generic DAAs from India of sofosbuvir, sofosbuvir/ledipasvir, sofosbuvir/velpatasvir, sofosbuvir/daclatasvir and, more recently, sofosbuvir/velpatasvir/ voxilaprevir. Importantly, at present no DAAs are registered by the South African Health Professions Regulation Authority (SAHPRA), and as such, in treating all patients, named patient approvals via Section 21 certificates were obtained. Treatment protocols followed existing American Association for the Study of Liver Disease, European Association for the Study of Liver Disease and local treatment guidelines. In principle, patients had 12 weeks of therapy with ribavirin added to treatment regimens of sofosbuvir/ledipasvir, sofosbuvir/daclatasvir or sofosbuvir/velpatasvir in those with cirrhosis. In clinically problematic patients likely to be intolerant of ribavirin, treatment was extended to 24 weeks without ribavirin. We performed a per protocol analysis of our experience in the first 2.5 years of using DAA therapies.

\section{Ethics approval}

The study was approved by the University of Cape Town Human Research Ethics Committee (ref. no. R045/2014).

\section{Statistical analysis}

All values are expressed as medians and interquartile ranges (IQRs) for continuous variables. Baseline and on-treatment data were summarised using standard descriptive characteristics. Where appropriate, differences between qualitative parameters were explored using the Wilcoxon rank-sum test. Statistical analysis was performed using Statistica version 13.5.0.17 (Dell, USA).

\section{Results}

Table 1 shows the baseline demographics of the first 210 patients who completed DAA therapy in an ongoing treatment programme. Almost two-thirds were men, who were significantly younger than women at the time of treatment (median age 50 years v. 58 years; $p=0.0018$ ). The GT distribution reaffirms the pan-genotypic nature of HCV in SA, with GTs 1 and 5 predominating (45\% and 20\%, respectively). However, a significant proportion, 17\%, were GT-4 infected. These patients were mostly black (47\%), with more than half originating from other countries in sub-Saharan Africa, including Rwanda, the Democratic Republic of the Congo (DRC), Cameroon, Malawi and Ethiopia. Overall, almost $40 \%$ of patients had advanced fibrosis (F3) or cirrhosis (F4) at baseline. In addition, $13 \%$ of patients were treatment experienced and 19\% were HIV co-infected, with 4 patients (2\%) HBV-HCV co-infected. Of those who were cirrhotic, $12 \%$ were Childs-Pugh class B or C. The likely mode of acquisition of hepatitis C (Table 1) is in keeping with known risk factors. Notably, no clear risk factor was identified in $26 \%$ of patients. Table 2 shows the diversity of HCV GT subtypes, with stark diversity noted in GTs 2 and 4 . Table 3 confirms the wide array of DAA regimens used, although NS5A/NS5B combination therapies predominated. These included sofosbuvir in combination with either daclatasvir, ledipasvir or velpatasvir, with the majority of patients accessing generic medicines. The overall SVR12 was $96 \%$. When those who 


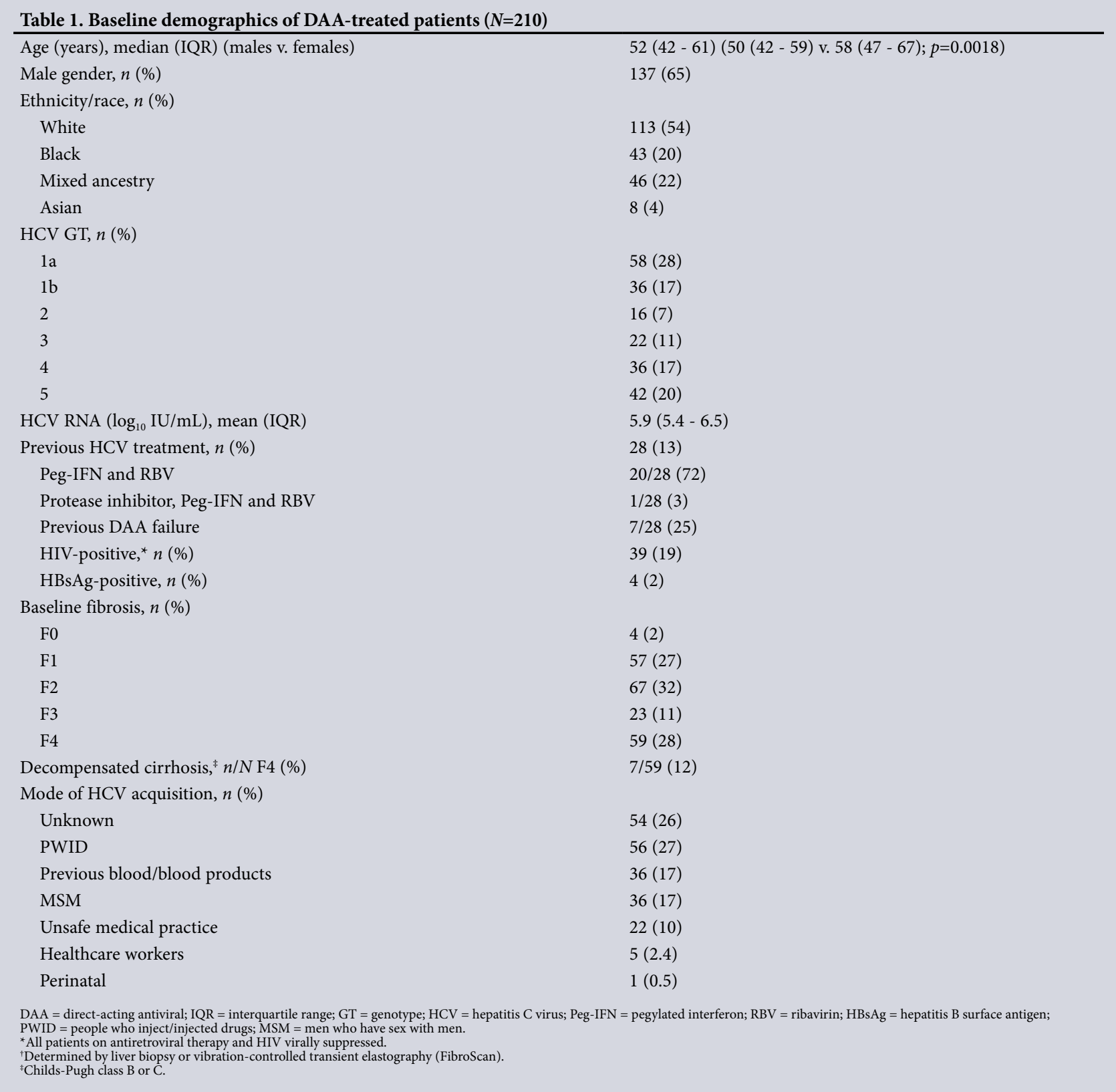

failed sofosbuvir/ribavirin (a combination no longer used for nonGT-2 patients) were excluded from this calculation, the overall SVR12 was 98\%. Looking specifically at GTs 1 b, 2 and 5, the SVR was $100 \%$. Notably, a non-SVR was observed in patients with GTs 3 and 4 , the majority GT- 4 and in particular subtype $4 \mathrm{r}$ (Table 4 ). In keeping with the efficacy of DAA therapy, $84 \%$ of patients were virally undetectable and below the lower level of quantification at 4 weeks of therapy. Paralleling this was a significant and rapid normalisation of serum ALT at week 4, with further improvement at the end of treatment $(p<0.0001)$. Of note, a significant decline in serum AFP from baseline to the end of treatment $(p<0.0001)$, a phenomenon not typically reported, was observed (Table 5).

\section{Discussion}

For the first time, we report local experience with DAA therapy for hepatitis C. As anticipated, key populations such as PWID and MSM constituted high proportions of our cohort (27\% and
17\%, respectively). Furthermore, despite a historically safe blood/ blood products supply system, haemophiliacs and those who received blood/blood products before 1992 were at risk, with $17 \%$ of our cohort sharing this as a risk factor. ${ }^{[16-19]}$ Additionally, we have demonstrated that unsafe medical practices are a risk factor (10\%). In a significant proportion of patients $(26 \%)$ no clear mode of acquisition was identified. In this group, black African patients were most likely to not have a clearly identifiable acquisition risk factor $(p<0.00001)$. Reasons for this finding are entirely unclear and may suggest exposure to unsafe medical or possibly traditional practices as a potential risk factor for $\mathrm{HCV}$ transmission. These factors certainly warrant further study to guide screening policies in SA and subSaharan Africa as a whole.

Globally, of the 71 million patients chronically infected with HCV, approximately half have HCV GTs other than GT-1. ${ }^{[20]}$ SA is a multi-genotypic country, with two studies confirming that GTs 1 and 5 were most prevalent and GTs 2,3 and 4 also prevalent. ${ }^{[1,22]}$ 


\begin{tabular}{ll} 
Table 2. Hepatitis C GT subtype diversity & \\
\hline GT subtype & $\boldsymbol{n}(\%)$ \\
\hline GT-1 $(N=94)$ & \\
1a & $58(62)$ \\
1b & $36(38)$ \\
GT-2 $(N=16)$ & \\
2a & $2(13)$ \\
2b & $3(19)$ \\
2c & $9(56)$ \\
2d & $1(6)$ \\
2q & $1(6)$ \\
GT-3 $(N=22)$ & \\
3a & $22(100)$ \\
GT-4 $(N=36)$ & \\
$4 \mathrm{a}$ & $3(8)$ \\
$4 \mathrm{~b}$ & $2(6)$ \\
$4 \mathrm{c}$ & $1(3)$ \\
$4 \mathrm{~d}$ & $5(14)$ \\
$4 \mathrm{c} / \mathrm{d}$ & $2(6)$ \\
$4 \mathrm{k}$ & $7(19)$ \\
$4 \mathrm{q}$ & $1(3)$ \\
$4 \mathrm{r}$ & $8(22)$ \\
$4 \mathrm{v}$ & $3(8)$ \\
4 (no subtype) & $4(11)$ \\
GT-5 (N=42) & \\
$5 \mathrm{a}$ & $42(100)$ \\
GT = genotype. & \\
&
\end{tabular}

\section{Table 3. DAA regimens $(N=206)$}

\begin{tabular}{ll}
\hline & $\boldsymbol{n}(\%)$ \\
\hline Sofosbuvir/ribavirin & $10(5)$ \\
Partitaprevir, ritonavir, ombitasvir + ribavirin & $8(4)$ \\
Partitaprevir, ritonavir, ombitasvir, dasabuvir + & $15(7)$ \\
ribavirin & \\
Sofosbuvir/daclatasvir ${ }^{*}$ & $75(36)$ \\
$\quad+$ ribavirin ${ }^{\dagger}$ & $12 / 75(16)$ \\
Sofosbuvir/ledipasvir ${ }^{*}$ & $80(38)$ \\
$\quad+$ ribavirin ${ }^{\dagger}$ & $15 / 80(19)$ \\
Sofosbuvir/velpatasvir & \\
Sofosbuvir/velpatasvir/voxilaprevir & $15(7)$ \\
Sofosbuvir/simeprevir/ribavirin & $5(2)$ \\
DAA = direct-acting antiviral. & $2(1)$ \\
${ }^{*}$ Exclusively generic therapy. & \\
${ }^{*}$ '63\% received brand-name therapy, $37 \%$ one of two generics. & \\
&
\end{tabular}

We noted a similar GT distribution, although marked GT-4 subtype diversity was also observed. Most GT-4 subtype diversity is observed in sub-Saharan Africa, with GT-4r, for example, frequently found in the DRC and Rwanda. ${ }^{[23]}$ Most of our black GT-4 patients originated from these and other African countries, explaining our observed diversity. Other unusual subtypes, e.g. GT-2c or GT-4d, clustered in a number of individuals with similar HCV transmission risk. These occurred exclusively in MSM, strongly supporting potential network transmission. Rapidly linking these patients to treatment is crucial in breaking the network of transmission. ${ }^{[24]}$

Our SVR rate of 96\%, in keeping with existing trial as well as realworld experience data, is encouraging. ${ }^{[25]}$ When we excluded our failed sofosbuvir/ribavirin patients from this assessment, SVR was

\section{Table 4. Outcomes of DAA-treated patients $(N=210)$}

\begin{tabular}{|c|c|}
\hline & $n(\%)$ \\
\hline \multicolumn{2}{|l|}{ HCV RNA <LLOQ during treatment period } \\
\hline At week 4 & $176(84)$ \\
\hline At week 12 & $208(99)$ \\
\hline At least 12 weeks after EOT (SVR) & $202(96)$ \\
\hline \multicolumn{2}{|l|}{ SVR according to GT } \\
\hline la & $57 / 58(98)$ \\
\hline $1 b$ & $36 / 36(100)$ \\
\hline 2 & $16 / 16(100)$ \\
\hline 3 & $20 / 22(91)$ \\
\hline $4^{*}$ & $31 / 36(86)$ \\
\hline 5 & $42 / 42(100)$ \\
\hline \multicolumn{2}{|l|}{ SVR per treatment regimen } \\
\hline Sofosbuvir/ribavirin & $8 / 10(80)$ \\
\hline Partitaprevir, ritonavir, ombitasvir + ribavirin & $7 / 8(88)$ \\
\hline $\begin{array}{l}\text { Partitaprevir, ritonavir, ombitasvir, dasabuvir + } \\
\text { ribavirin }\end{array}$ & $15 / 15(100)$ \\
\hline Sofosbuvir/daclatasvir ${ }^{\dagger}$ & 73/75 (97) \\
\hline Sofosbuvir/ledipasvir & $78 / 80(98)$ \\
\hline Sofosbuvir/velpatasavir & $15 / 15(100)$ \\
\hline Sofosbuvir/velpatasavir/voxilaprevir & $4 / 5(80)$ \\
\hline \multicolumn{2}{|l|}{ Virological failure ( $N=8$ without SVR) } \\
\hline During treatment & $2 / 8(25)$ \\
\hline After treatment & $6 / 8(75)$ \\
\hline Adverse events ${ }^{\ddagger}$ & $22(10)$ \\
\hline Discontinued therapy because of adverse events & 0 \\
\hline \multicolumn{2}{|c|}{ 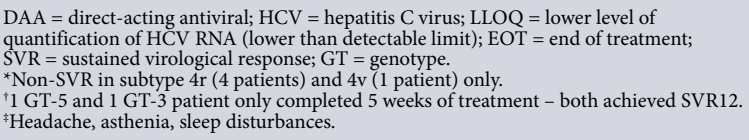 } \\
\hline
\end{tabular}

98\%. Sofosbuvir/ribavirin was an early therapy that demonstrated an improvement well above Peg-IFN/ribavirin therapy and offered the first 'all-oral' option for patients. However, overall SVR rates (except for GT-2) fell short of the now accepted $>90 \%$ benchmark SVR rate expected of DAA therapy, and this combination has now mostly been abandoned in favour of more efficacious combinations. It remains a reasonable option for GT-2, but patients need to tolerate 12 weeks of ribavirin. If they are unable to do so, ribavirin-free options exist. ${ }^{[26]}$

When contextualised in terms of the baseline demographics of our patients, our outcomes confirm treatment efficacy in what were previously termed 'difficult-to-treat' patients, with almost $40 \%$ having advanced fibrosis or cirrhosis. Of the $28 \%$ with cirrhosis, $12 \%$ had Childs-Pugh class B or C disease. In addition, almost $20 \%$ were HIV co-infected, and $13 \%$ were treatment experienced. All these factors would have significantly affected SVR rates in the Peg-IFN/ribavirin era of therapy, with some ineligible for therapy. DAA therapy now negates these factors, and very few patients, if any, pose clinical challenges to therapy. Furthermore, therapy was well tolerated, with no patients discontinuing therapy due to adverse effects. In keeping with most trial data, $10 \%$ reported a side-effect, mostly minor and including headache and mild sleep disturbances. These typically occur early in therapy and usually abate. ${ }^{[27]}$

Serum transaminases significantly declined between week 4 and the end of treatment and mirrored the rapid loss of HCV RNA. Another observation was the significant decline in serum AFP, particularly 
Table 5. Laboratory parameters at baseline and on treatment

\begin{tabular}{lll}
\hline & Median (IQR) & $p$-value \\
\hline ALT $(\mathrm{U} / \mathrm{L})$ & & $<0.0001$ \\
Baseline & $74(41-124)$ & \\
At week 4 & $22(15-28)$ & \\
At end of treatment & $18(14-26)$ & $<0.0001$ \\
AFP $(\mu \mathrm{g} / \mathrm{L})$ & $5.4(3-13)$ & \\
Baseline & $3.7(2.7-7.5)$ & \\
At end of treatment & & \\
ALT = alanine transaminase; AFP = alpha-fetoprotein; IQR = interquartile range. &
\end{tabular}

in patients with cirrhosis. This phenomenon has been described previously, and it is an independent predictor of declining HCC risk in patients with advanced fibrosis or cirrhosis. ${ }^{[28-30]}$ Importantly, patients with cirrhosis, despite SVR, warrant lifelong HCC surveillance.

Focusing specifically on GTs, SVR 12 was $100 \%$ in GTs $1 \mathrm{~b}, 2$ and 5. A single patient with GT-1a failed therapy and was successfully retreated with a sofosbuvir/simeprevir/ribavirin regimen. SVR was not achieved in 2 patients with GT-3a. One was treated with sofosbuvir/ribavirin and the other with sofosbuvir/daclatasvir. Problems with sofosbuvir/ribavirin are noted above, but failure to achieve SVR in a second patient on sofosbuvir/daclatasvir was probably due to the presence of cirrhosis as well a particularly problematic RAS mutation $(Y 93 H)$. This mutation poses potential resistance to NS5A inhibitors, especially in GT-3-infected patients. ${ }^{[31]}$ Concerningly, several treatment failures occurred in GT-4 patients, including subtypes $4 \mathrm{r}$ (4 patients) and $4 \mathrm{v}$ ( 1 patient). Our experience, together with newly published data from Rwanda and France, ${ }^{[32,33]}$ suggests that GT-4r has a reduced sensitivity to NS5A inhibitors, especially ledipasvir. In the Rwanda study, ${ }^{[32]}$ only $54 \%$ of GT- $4 \mathrm{r}$ patients achieved SVR. Furthermore, the latest data suggest that the wide diversity of GT-4 and the array of baseline RAS mutations pose a therapeutic challenge that would disproportionally affect subSaharan Africa, given GT-4 and its subtype diversity prevalence. ${ }^{[34]}$ A better understanding and further study of what regimen is best to treat GT-4r and other potentially problematic subtypes is needed.

Most of our patients were treated with sofosbuvir-based therapy in combination with one of three NS5A inhibitors, viz. daclatasvir, ledipasvir or velpatasvir. Given the lack of registration of DAAs by the SAHPRA to date, cost issues and the lack of initial availability of originator therapies, generics were sourced from Indian-based generic manufacturers. In our cohort, originator and generic therapies were equally effective. Once originator drug products are registered, generic availability, as a Section 21 therapy, will cease. This will pose a significant barrier to potential access for many if costs are not negotiated with manufacturers by government, in advance of registration. In particular, price parity between public and private sector pricing must be sought. A price differential cannot be the norm if the elimination of viral hepatitis is the objective in SA, as it is globally. This objective, as per the World Health Organization 2016 adopted global health sector strategy policy, aims to eliminate viral hepatitis by $2030 .{ }^{[35]} \mathrm{SA}$ adopted its own costed plan in this regard in 2018. ${ }^{[36]}$ We recommend that SA utilise pangenotypic DAA therapies for its strategic plan through sofosbuvir plus daclatasvir or velpatasvir. This strategy omits the potential need for genotyping in the treatment cascade, allowing for rapid linkage to care. Routine HCV Sanger sequencing in DAA-naive patients prior to treatment is not recommended.

Underpinning the gigantic therapeutic advance of DAA therapy is the number of people who have been linked to care. Over a $>10$-year period, our clinic treated 77 patients with Peg-IFN/ribavirin with an overall SVR rate of $75 \%$. This was achieved through careful patient selection by limiting access to those with difficult-to-treat clinical and virological characteristics. In 2.5 years, three times more people were treated with excellent outcomes, many of them previously regarded as difficult to treat.

\section{Conclusions}

DAA therapy has fundamentally changed the approach to the management of hepatitis $\mathrm{C}$ and provided impetus towards the elimination of hepatitis C. However, many challenges remain, including access and the need for new models and approaches to the delivery of care to key populations. The current model of care based at a tertiary-level specialist clinic is not feasible for the upscaling of treatment. What we have demonstrated is that even in our resourceconstrained environment, linking patients to this curative therapy is possible and has the potential to be expanded to all levels of care.

\section{Declaration. None.}

Acknowledgements. We express gratitude to our patients with hepatitis $C$ who have embraced the new generation of therapy, ridding them of a potentially deadly disease. More patients need access! We are especially grateful to those who have provided therapy, viz. MSF South Africa, the AbbVie compassionate use programme and our Drug and Therapeutics Committee and Pharmacy at Groote Schuur Hospital, for their progressiveness and recognition of the need and value of this therapy being made available well before registration has occurred in South Africa. Author contributions. MWS drafted the manuscript. All authors contributed to the editing process and were involved in patient care.

Funding. None.

Conflicts of interest. None.

1. Sonderup MW, Afihene M, Ally R, et al. Hepatitis C in sub-Saharan Africa: The current status an recommendations for achieving elimination by 2030. Lancet Gastroenterol Hepatol 2017;2(12):910919. https://doi.org/10.1016/S2468-1253(17)30249-2

2. Polaris Observatory HCV Collaborators. Global prevalence and genotype distribution of hepatitis $\mathrm{C}$ virus infection in 2015: A modelling study. Lancet Gastroenterol Hepatol 2017;2(3):161-176. https:// virus infection in 2015: A modelling study.

Scheibe A, Young K, Moses L, et al. Understanding hepatitis B, hepatitis C and HIV among people Scheibe A, Young K, Moses L, et al. Understanding hepatitis B, hepatitis C and HIV among people
who inject drugs in South Africa: Findings from a three-city cross-sectional survey. Harm Reduct J who inject drugs in South Africa: Findings from a three-cily

4. Vermeulen M, Swanevelder R, Chowdhury D, et al. Use of blood donor screening to monito prevalence of HIV and hepatitis B and C viruses, South Africa. Emerg Infect Dis 2017;23(9):15601563. https://doi.org/10.3201/eid2309.161594

5. Gogela NA, Sonderup MW, Rebe K, Chivese T, Spearman CW. Hepatitis C prevalence in HIV-infected heterosexual men and men who have sex with men. S Afr Med J 2018;108(7):568-572. https://doi org/10.7196/SAMJ.2018.v108i7.13041

6. Semugoma NP, Rebe K, Sonderup MW, et al. Hepatitis C: A South African literature review and results from a burden of disease study among a cohort of drug-using men who have sex with men in Cape Town, South Africa. S Afr Med J 2017;107(12):1116-1120. https://doi.org/10.7196/SAMJ.2017. v107i12.12623

7. Choo QL, Kuo G, Weiner AJ, Overby LR, Bradley DW, Houghton M. Isolation of a cDNA clone derived from a blood-borne non-A, non-B viral hepatitis genome. Science 1989;244(4902):359-362. https:// doi.org/10.1126/science. 2523562 
8. Fried MW, Shiffman ML, Reddy KR, et al. Peginterferon alfa-2a plus ribavirin for chronic hepatitis C virus infection. N Engl J Med 2002;347(13):975-982. https://doi.org/10.1056/NEJMoa020047

9. Poynard T, McHutchison J, Manns M, et al. Impact of pegylated interferon alfa-2b and ribavirin on liver fibrosis in patients with chronic hepatitis C. Gastroenterology 2002;122(5):1303-1313. https:// doi.org/10.1053/gast.2002.33023

10. Bartenschlager R, Lohmann V. Replication of hepatitis C virus. J Gen Virol 2000;81(Pt 7):1631-1648. https://doi.org/10.1099/0022-1317-81-7-1631

1. Bartenschlager R, Kaul A, Sparacio S. Replication of the hepatitis $C$ virus in cell culture. Antiviral Res 2003;60(2):91-102. https://doi.org/10.1016/j.antiviral.2003.08.016

12. Afdhal N, Reddy KR, Nelson DR, et al. Ledipasvir and sofosbuvir for previously treated HCV genotype 1 infection. N Engl J Med 2014;370(16):1483-1493. https://doi.org/10.1056 NEJMoa1316366

13. Geddawy A, Ibrahim YF, Elbahie NM, Ibrahim MA. Direct acting anti-hepatitis C virus drugs: Clinical pharmacology and future direction. J Transl Intern Med 2017:5(1):8-17, https://dol. org/10.1515/jim-2017-0007

14. Holmes JA, Rutledge SM, Chung RT. Direct-acting antiviral treatment for hepatitis C. Lancet 2019;393(10179):1392-1394. https://doi.org/10.1016/s0140-6736(18)32326-2

15. Sonderup MW, Spearman CW. Access to generic medicines for hepatitis $C$ in South Africa: $A$ journey of discovery. Med Access @ Point Care 2017;1(1):e6-e9. https://doi.org/10.5301/maapoc.0000002

16. Vermeulen M, Swanevelder R, Chowdhury D, et al. Use of blood donor screening to monitor prevalence of HIV and hepatitis B and C viruses, South Africa. Emerg Infect Dis 2017;23(9):15601563. https://doi.org/10.3201/eid2309.161594

17. Fang CT, Field SP, Busch MP, Heyns A du P. Human immunodeficiency virus-1 and hepatitis $C$ virus RNA among South African blood donors: Estimation of residual transfusion risk and yield of nucleic acid testing. Vox Sang 2003;85(1):9-19. https://doi.org/10.1046/j.1423-0410.2003.00311.x

18. Soni PN, Robson SC, Kirsch RE, Simjee AE. Anti-hepatitis C antibody screening at the Natal Blood Transfusion Service. S Afr Med J 1993;83(3):218.

19. Tucker TJ, Voigt M, Bird A, et al. Hepatitis $\mathrm{C}$ virus infection rate in volunteer blood donors from the Western Cape - comparison of screening tests and PCR. S Afr Med J 1997;87(5):603-605.

20. Polaris Observatory HCV Collaborators. Global prevalence and genotype distribution of hepatitis $\mathrm{C}$ virus infection in 2015: A modelling study. Lancet Gastroenterol Hepatol 2017:2(3):161-176. https:/ doi.org/10.1016/S2468-1253(16)30181-9 L

21. Prabdial-Sing N, Chirwa T, Thaver J, et al. Hepatitis C genotype distribution in patient and blood donor samples in South Africa for the period 2008 - 2012. J Viral Hepat 2016;23(11):881-888. https://doi.org/10.1111/jwh. 12571

22. Smuts HE, Kannemeyer I. Genotyping of hepatitis C virus in South Africa. J Clin Microbiol 1995;33(6):1679-1681

23. Davis C, Mgomella GS, da Silva Filipe A, et al. Highly diverse hepatitis C strains detected in subSaharan Africa have unknown susceptibility to direct-acting antiviral treatments. Hepatology 2019;69(4):1426-1441. https://doi.org/10.1002/hep.30342
24. Scott N, Stoové M, Wilson DP, et al. Eliminating hepatitis $\mathrm{C}$ virus as a public health threat among HIVpositive men who have sex with men: A multi-modelling approach to understand differences in sexual positive men who have sex with men: A multi-modelling approach to understand differ
risk behaviour. J Int AIDS Soc 2018;21(1):e25059. https://doi.org/10.1002/jia2.25059

25. Hézode C. Treatment of hepatitis C: Results in real life. Liver Int 2018:38(S1):21-27. https://doi org/10.1111/liv.13638.

26. Zeuzem S, Dusheiko GM, Salupere R, et al. Sofosbuvir and ribavirin in HCV genotypes 2 and $3 . \mathrm{N} \mathrm{Engl}$ J Med 2014;370(21):1993-2001. https://doi.org/10.1056/NEJMoa1316145

27. Banerjee D, Reddy KR. Review article: Safety and tolerability of direct-acting anti-viral agents in the new era of hepatitis C therapy. Aliment Pharmacol Ther 2016;43(6):674-696. https://doi.org/10.1111/ apt.13514

28. Di Bisceglie AM, Sterling RK, Chung RT, et al. Serum alpha-fetoprotein levels in patients with advanced hepatitis C: Results from the HALT-C Trial. J Hepatol 2005;43(3):434-441. https://doi. org/10.1016/j.jhep.2005.03.019

29. Asahina $Y$, Tsuchiya K, Nishimura T, et al. Alpha-fetoprotein levels after interferon therapy and risk of hepatocarcinogenesis in chronic hepatitis C. Hepatology 2013;58(4):1253-1262. https://doi. org/10.1002/hep.26442

30. Fouad R, Elsharkawy A, Abdel Alem S, et al. Clinical impact of serum alpha-fetoprotein and its relation on changes in liver fibrosis in hepatitis $\mathrm{C}$ virus patients receiving direct-acting antivirals. Eur $\mathrm{J}$ Gastroenterol Hepatol 2019;31(9):1129-1134. https://doi.org/10.1097/MEG.0000000000001400

31. Smith D, Magri A, Bonsall D, et al. Resistance analysis of genotype 3 hepatitis C virus indicates subtypes inherently resistant to nonstructural protein 5A inhibitors. Hepatology 2019;69(5):18611872. https://doi.org/10.1002/hep.29837

32. Gupta N, Mbituyumuremyi A, Kabahizi J, et al. Treatment of chronic hepatitis C virus infection in Rwanda with ledipasvir-sofosbuvir (SHARED): A single-arm trial. Lancet Gastroenterol Hepatol 2019;4(2):119-126. https://doi.org/10.1016/S2468-1253(18)30382-0

33. Fourati S, Rodriguez C, Hezode C, et al. Frequent antiviral treatment failures in patients infected with hepatitis C virus genotype 4, subtype 4r. Hepatology 2019;69(2):513-523. https://doi.org/10.1002/ hep. 30225

34. Davis C, Mgomella GS, da Silva Filipe A, et al. New highly diverse hepatitis C strains detected in sub-Saharan Africa have unknown susceptibility to direct-acting antiviral treatments. Hepatology 2019;69(4):1426-1441. https://doi.org/10.1002/hep.30342

35. Thomas DL. Global elimination of chronic hepatitis. N Engl J Med 2019;380(21):2041-2050. https:// doi.org/10.1056/NEJMra1810477

36. Hecht R, Hiebert L, Spearman WC, et al. The investment case for hepatitis B and C in South Africa: Adaptation and innovation in policy analysis for disease program scale-up. Health Policy Plan 2018;33(4):528-538. https://doi.org/10.1093/heapol/czy018

Accepted 5 December 2019 